\title{
Molecular Investigation of a Fungemia Outbreak Due to Candida parapsilosis in an Intensive Care Unit
}

Murat Dizbay ${ }^{1}$, Ayse Kalkanci², Busra Ergut Sezer ${ }^{1}$, Firdevs Aktas ${ }^{1}$, Sibel Aydogan², Isil Fidan², Semra Kustimur ${ }^{2}$ and Takashi Sugita $^{3}$ ${ }^{1}$ Gazi University Faculty of Medicine, Department of Clinical Microbiology and Infectious Diseases and Infection Control Committee; ${ }^{2}$ Department of Microbiology; Ankara, Turkey; ${ }^{3}$ Meiji Pharmaceutical University, Department of Microbiology Kiyose; Tokyo, Japan

\begin{abstract}
We investigated a nosocomial cluster of four Candida parapsilosis fungemia episodes that occurred in a neurological intensive care unit over a two-week period. The four infected patients had received parenteral nutrition through central lines, and all four had catheter-related candidemia. All of the isolates were susceptible to all of the antifungals tested, including amphotericin B, fluconazole, voriconazole, and caspofungin. They had strictly related fingerprints, based on randomly amplified polymorphic DNA analysis. Additional DNA sequencing data revealed that they were same strain. Although no isolate of Candida parapsilosis was recovered from other clinical, surveillance, or environmental samples, nosocomial spread of this yeast ceased, following the reinforcement of infection-control measures. Candida parapsilosis may require an intravascular foreign body to cause fungemia, but this outbreak shows that it can be transmitted nosocomially and can cause epidemics.

Key-Words: Candidemia, Candida parapsilosis, nosocomial outbreak, intensive care unit, RAPD, DNA sequencing.
\end{abstract}

Candida species are the third most common cause of nosocomial bloodstream infections (BSI) in intensive care units, with a $47.1 \%$ crude mortality rate in the United States [1]. Candidemias have been found to be associated with increasing mortality, length of hospital stay and hospital costs [2,3]. Among the Candida species identified to date, Candida albicans still ranks first, but recent studies show a shift towards increasing prevalence of non-albicans Candida spp. [4-6]. This change has been attributed partly to the widespread use of antifungal agents and to an increasing number of immunocompromised hosts; however, it may also reflect an increasing awareness that non-albicans Candida isolates are important opportunistic pathogens. In a neurological intensive care unit (NICU) of our hospital, we observed a nosocomial cluster of fungemia due to Candida parapsilosis that affected four patients. We retrospectively examined the characteristics of the candidemia episodes in these patients and employed molecular techniques to determine whether the fungal isolates from these patients were of clonal origin.

\section{Material and Methods}

Patients

Four cases of $C$. parapsilosis fungemia were identified within a two-week period in an NICU at the University Hospital of Gazi, a 1,000-bed tertiary care hospital, in Ankara, Turkey. The NICU has one patient room (a total of seven beds), mainly occupied by elderly patients affected by stroke, cerebrovascular disease, or intracranial hemorrhage. At the time the candidemia cases were detected, the four affected patients were in same room. Their periods of hospitalization overlapped, and they were all cared for by NICU staff members.

Received on 12 April 2008; revised 20 September 2008.

Address for correspondence: Dr. Isil Fidan. Gazi University, Faculty of Medicine, Department of Medical Microbiology. Dekanlik Binasi 2.Kat Besevler/ANKARA 06500, TURKEY. Phone: +90 312 2024626. Fax: +90 312 4358632. E-mail: isilfidan@yahoo.com.

The Brazilian Journal of Infectious Diseases

2008;12(5):395-399. (C) 2008 by The Brazilian Journal of Infectious Diseases and Contexto Publishing. All rights reserved.
The clinical characteristics of the four patients are summarized in Table 1.

Active surveillance program is routinely performed for infection control in the NICU. After the second case of $C$. parapsilosis fungemia was diagnosed, molecular epidemiological investigations were initiated. Physicians and nursing staff of the intensive care unit were screened for oral and hand carriage of Candida spp. Extensive sampling was undertaken from fomites and other environmental sources of the ward (floors, disinfectant solutions, multi-dose vials, infusion pumps, commercially prepared parenteral nutrition bags, and other medical equipments), and cultures were made. Finally, compliance with standard infection control measures, including rigorous hand-washing, was emphasized.

Strains

All of the C. parapsilosis strains were isolated from the cultures of blood specimens or central venous catheter (CVC) tips from the four patients. The yeastlike organisms were identified according to their morphological characteristics, biochemical profiles obtained with the ID 32C kit (bioMérieux, France), assimilation of six additional sugars, and growth morphology on corn meal agar plates.

\section{Antifungal Susceptibility Tests}

Antifungal susceptibility testing for amphotericin B, fluconazole, voriconazole and caspofungin was performed for each of the strains by the microdilution method, according to the Clinical Laboratory Standards Institution (CLSI, formerly NCCLS) reference microdilution method documented in M27A2 [7].

Randomly Amplified Polymorphic DNA and DNA Sequencing

All of the $C$. parapsilosis isolates were typed by randomly amplified polymorphic DNA (RAPD). Two oligonucleotide primers, forward 5'-ATG ACT CCA GCT GGT TC-3' and reverse 5'- TAG ATC AAG AAT GCA, were used for RAPD analysis. Briefly, approximately 10 ng Candida DNA was added to a $0.5 \mathrm{~mL}$ microfuge tube containing $20 \mathrm{pmol}$ 
oligonucleotide primer, $250 \mu \mathrm{M}$ each of dATP, dTTP, dCTP and dGTP, $3 \mathrm{mM} \mathrm{MgCl}$, $2.5 \mathrm{U}$ Taq DNA polymerase and 10X buffer in a final volume of $25 \mu \mathrm{L}$ (all of the chemicals were obtained from Metis Biyoteknoloji, Turkey). The amplification procedure was performed with an initial denaturation step at $94^{\circ} \mathrm{C}$ for $1 \mathrm{~min}$, followed by 45 cycles of, $30 \mathrm{~s}$ at $94^{\circ} \mathrm{C}, 60 \mathrm{~s}$ at $42^{\circ} \mathrm{C}$, and $90 \mathrm{~s}$ at $72^{\circ} \mathrm{C}$, with a final extension at $72^{\circ} \mathrm{C}$ for $7 \mathrm{~min}$ in a thermal cycler (Hybaid, UK). The products were separated in $2 \%(\mathrm{w} / \mathrm{v})$ agarose gels containing $0.5 \mu \mathrm{g}$ ethidium bromide $\mathrm{mL}^{-1}$ and viewed on a UV transluminator.

DNA sequencing was additionally performed for identifying C. parapsilosis. The D1/D2 regions of 26S rDNA in the rRNA gene were sequenced directly from PCR products using the primer pair NL1 (GCA TAT CAA TAA GCG GAG GAAAAG) and NL4 (GGT CCG TGT TTCAAG ACG G). The PCR products were sequenced using an ABI 310 DNA sequencer and a BigDye Terminator Cycle Sequencing Ready Reaction kit (Perkin-Elmer), according to the manufacturer's instructions. The sequence data were analyzed using the National Center for Biotechnology Information (Bethesda, Md., USA) BLAST system (available at http:// www.ncbi.nlm.nih.gov/BLAST/).

\section{Results}

Strains

Ten samples obtained from blood specimens or catheter tips removed from the four patients were culture-positive for yeast organisms. All isolates showed identical phenotypic characteristics and were identified as $C$. parapsilosis. The quality of identification of $C$. parapsilosis with ID 32C was acceptable in all strains. Yeasts were identified morphologically as $C$. parapsilosis on corn meal agar plates.

\section{Antifungal Susceptibility Tests}

All isolates were susceptible to amphotericin B (MIC, $0.125 \mu \mathrm{g} / \mathrm{mL}$ ), fluconazole (MIC, $0.125 \mu \mathrm{g} / \mathrm{mL}$ ), voriconazole (MIC, $0.250 \mu \mathrm{g} / \mathrm{mL}$ ) and caspofungin (MIC, $0.062 \mu \mathrm{g} / \mathrm{mL}$ ).

\section{RAPD Analysis}

RAPD analysis was performed for further characterization of $C$. parapsilosis isolates. The overall RAPD profiles of the clinical isolates were very similar to each other, but very different from the profiles of the C. parapsilosis ATCC 22019 reference strain (Figure 1). These results suggest that the isolates from the four patients were derived from the same strain. Candida parapsilosis was not isolated from the surveillance cultures of the healthcare workers or from environmental sources. The review of infection control procedures used in the NICU and in the pharmacy did not show any relapse.

\section{DNA Sequences}

The DNA sequences of all the isolates completely matched to that of $C$. parapsilosis from the GenBank DNA database. Therefore, all the isolates were identified as a single strain of
C. parapsilosis. Clonal spread was confirmed by DNA sequencing results (Figure 2).

\section{Discussion}

Numerous outbreaks of systemic candidiasis in hospitalized patients have been reported $[2,4,8]$. Candida spp. was found to be responsible for outbreaks associated with parenteral nutrition, invasive devices, and intraoperative contamination, as well as cross-infection via the hands of hospital personnel or to extrinsic contamination of parenteral nutrition solutions that occurred during preparation or administration [3,5,6,8]. Since the hands of healthcare workers have been documented to be a reservoir of Candida spp., exogenous infection or cross-infection of the patients can be common $[9,10]$. Although an investigation was retrospectively carried out in each of these outbreaks, no definitive source of the fungal strains could be found.

Candida parapsilosis particularly affects critically ill neonates and ICU patients, likely because of its association with parenteral nutrition and central catheter lines [11,12]. The affinity of $C$. parapsilosis for foreign material is shown by infections [13]. Candida parapsilosis is increasingly responsible for hospital outbreaks, and the hands of healthcare workers can be the predominant environmental source. Total parenteral nutrition (TPN) solutions may promote C. parapsilosis adhesion and growth. Recently, biofilmforming potential was cited as a reason that patients with $C$. parapsilosis-infected catheters should have the device removed [14].

The prevalence of $C$. parapsilosis fungemia has increased over the years, and now, in some areas, $C$. parapsilosis is the second most common species found in patients with candidemia [15]. Risk factors for systemic infections with $C$. parapsilosis are prolonged neutropenia, aggressive chemotherapy, or use of broad spectrum antibacterial agents, and the alteration of local defenses by the obstruction or breakdown of the skin and mucosa. Epidemiological data obtained from fungemia patients revealed that non-albicans strains are gaining importance because of low-susceptibility rates to licensed antifungal drugs [16]. The reasons for the rising incidence of $C$. parapsilosis candidemia are not completely known, although indwelling venous catheters and parenteral nutrition have been recognized as specific risk factors $[4,17,18]$. Most experimental studies have indicated that the adherence of $C$. parapsilosis to acrylic surfaces is greater than that of C. albicans [19].

We identified four episodes of $C$. parapsilosis fungemia in four patients (mean age, 75.8 years) admitted to a NICU of a tertiary care hospital over a two-week period. All of the patients had neurological disease, had received parenteral nutrition solutions through a CVC, and had been cared for by personnel of the ICU during overlapping periods. All of the four patients had persistent CVC-related candidemia. All of the patients underwent CVC removal and received antifungal therapy following the diagnosis of candidemia. Fungemia 
Table 1. Clinical characteristics of four patients with nosocomially-transmitted Candida parapsilosis fungemia.

\begin{tabular}{|c|c|c|c|c|}
\hline Characteristic & Patient 1 & Patient 2 & Patient 3 & Patient 4 \\
\hline Age (years)/sex & 73/male & 79/female & 78/male & 73/male \\
\hline Underlying disease & Intracranial infarction & Cerebrovascular disease & Syncope & $\begin{array}{l}\text { Generalized } \\
\text { tonic-clonic seizure }\end{array}$ \\
\hline \multicolumn{5}{|c|}{ Potential risk factors for candidemia ${ }^{a}$} \\
\hline CVC & Yes & Yes & Yes & Yes \\
\hline Mechanic ventilation & Yes & Yes & Yes & Yes \\
\hline Bladder catheter & Yes & Yes & Yes & Yes \\
\hline Parenteral nutrition & Yes & Yes & Yes & Yes \\
\hline BSA & Yes & Yes & Yes & Yes \\
\hline VAP & Yes & No & Yes & Yes \\
\hline Diabetes mellitus & No & No & No & No \\
\hline Malignancy & No & No & No & No \\
\hline $\begin{array}{l}\text { No. of positive blood } \\
\text { cultures (culture site) }\end{array}$ & 3 (peripheral veins) & 2 (peripheral veins) & 4 (peripheral veins) & 1 (peripheral vein) \\
\hline \multicolumn{5}{|l|}{ Therapy } \\
\hline Antifungal therapy & $\begin{array}{l}\text { AMB, L-AMB, } \\
\text { Caspofungin }\end{array}$ & AMB & Fluconazole, & AMB \\
\hline Catheter removal & Yes & Yes & Yes & Yes \\
\hline Catheter-related candic & Yes & Yes & Yes & Yes \\
\hline \multicolumn{5}{|l|}{ Outcome } \\
\hline Candidemia & Cleared & Cleared & Failed & Cleared \\
\hline Clinical & Excitus & Recovery & Excitus & Recovery \\
\hline
\end{tabular}

CVC, central venous catheter; ND, not determined; AMB, amphotericin B; L-AMB, liposomal amphotericin B; VAP, ventilation associated

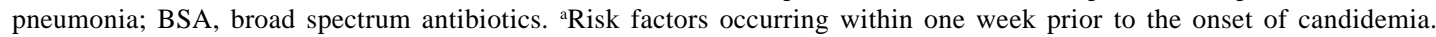

Figure 1. Random amplified polymorphic DNA fingerprints of Candida parapsilosis clinical isolates. Lanes 1 to 4 , isolates from the blood of patients, lane 5, C. parapsilosis clinical isolate, lane 6, C. parapsilosis ATCC 22019 reference strain. lane M, lambda HaeIII molecular size marker.

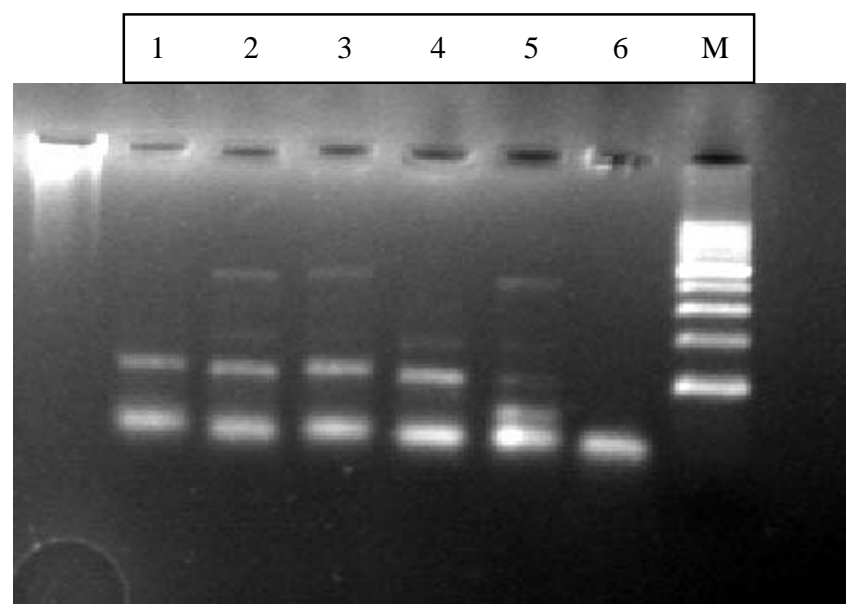

cleared in two of the patients, but two of them died later as a consequence of their underlying disease.

All of the C. parapsilosis strains isolated from blood samples were identified in our laboratory by morphological and biochemical methods that are used by many clinical laboratories. Recent studies have shown that fungal 26S rDNA and ITS region sequence data facilitate earlier and more reliable identification than phenotypic methods. Oligonucleotide primers based on the sequences of $18 \mathrm{~S}$ or $26 \mathrm{~S}$ rDNA have been designed for PCR and used in the detection of fungi. Unfortunately, these sophisticated molecular methods are available only in reference laboratories.

Molecular epidemiology methods are required for the demonstration of clonal relationships among isolates. The RAPD or arbitrarily primed polymerase chain reaction (APPCR) analysis is technically simple and often detects variations among Candida spp. isolates that are not discriminated with other typing methods. Though it has good discriminatory capacity, low cost and is easy to use, morphotyping has low reproducibility [20]. We used RAPD analysis to investigate the molecular epidemiology of blood isolates recovered from the patients hospitalized in the NICU of a tertiary care hospital. The clinical value of a single procedure, such as RAPD analysis, for determining both species and biotype is most clear in the area of molecular epidemiology. According to some authors, given the increase in nosocomial infections caused by Candida species, there is an urgent need for a rapid and simple procedure that would allow for the analysis of both the outbreaks and the incidence of person-to-person transmission of these organisms [21]. As such, more in-depth subsequent epidemiological analyses are required to more accurately clarify whether there are simply genetic similarities in a characteristic cloned population among these isolates or whether hospital 
Figure 2. Nucleotide sequence of the D1/D2 region of the large (26S) subunit rRNA genes.

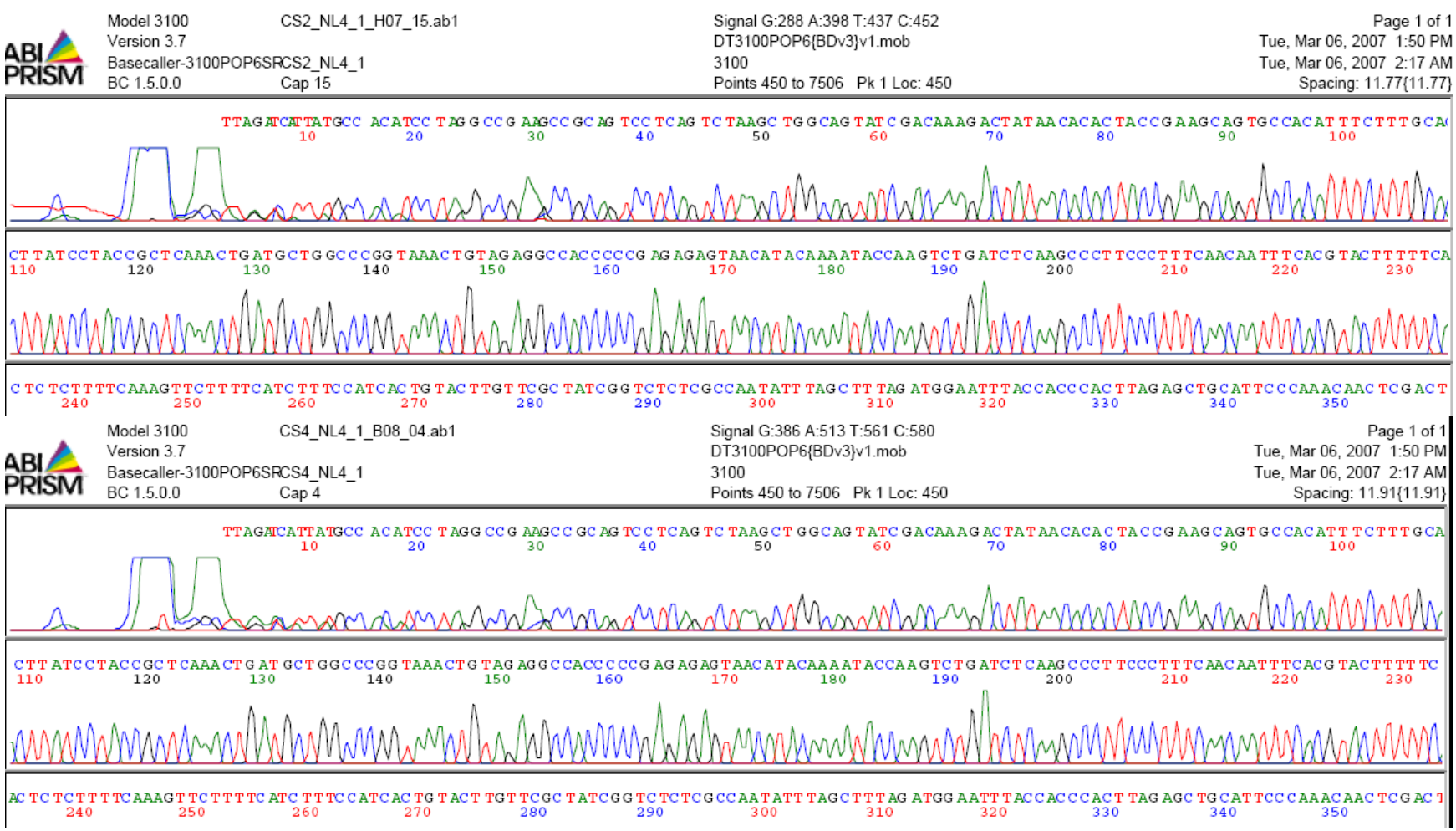

procedures help disseminate the agents among these patients through cross-infections. DNA sequencing is such method that can be used for both in the identification of species and the demonstration of clonal spread [22]. In our study, $C$. parapsilosis isolates obtained from the patients were identified as the same strain by direct DNA sequence analysis. The DNA examination results therefore suggest that $C$. parapsilsosis was transmitted to the patients in the hospital environment.

At the time of our investigation, we were unable to identify C. parapsilosis in any of the other clinical or environmental samples. Since patient screening was not carried out at hospital admission, and the cluster was only investigated retrospectively, it is difficult to determine whether these patients became infected from a common source within the hospital environment or if the yeast was transmitted from the first patient to the others. Although the outbreak strain was not isolated from the hands of the ward personnel during our retrospective investigation, the transmission of $C$. parapsilosis between patients and staff would seem likely. Indeed, the nosocomial spread of fungal infections ceased just after the standard infection control measures were reinforced in the ICU.

In conclusion, this outbreak shows the potential for nosocomial epidemic transmission of C. parapsilosis, an organism that may require an intravascular foreign body to cause fungemia. Investigation of an outbreak by molecular methods has diagnostic value when the primary source can not be found. This study proves the importance of a molecular approach for investigating suspected outbreaks in hospital survey.

\section{References}

1. Wisplinghoff H., Bischoff T., Tallent S.M., et al. Nosocomial bloodstream infections in US hospitals: analysis of 24,179 cases from a prospective nationwide surveillance study. Clin Infect Dis 2004;39:309-17.

2. Zaoutis T.E., Argon J., Chu J., Berlin J.A., Walsh T.J., Feudtner C. The epidemiology and attributable outcomes of candidemia in adults and children hospitalized in the United States: a propensity analysis. Clin Infect Dis 2005;41:1232-9.

3. Falagas M.E., Apostolou K.E., Pappas V.D. Attributable mortality of candidemia: a systematic review of matched cohort and case-control studies. Eur J Clin Microbiol Infect Dis 2006;25:419-25.

4. Barberino M.G., Silva N., Rebounças C., et al Evaluation of blood stream infections by Candida in three tertiary hospitals in Salvador, Brazil: A case-control study. Brazil J Infect Dis 2006;10:36-40.

5. Bakir M., Cerikcioglu, N., Barton R., Yagci A. Epidemiology of candidemia in a Turkish tertiary care hospital. APMIS 2006;111:601-10.

6. Warren D.K., Quadir W.W., Hollenbeak C.S., et al. Attributable cost of catheter-associated bloodstream infections among intensive care patients in a nonteaching hospital. Crit Care Med 2006;34:2243-4.

7. National Committee for Clinical Laboratory Standards (2002) Reference method for broth dilution antifungal susceptibility testing of yeasts; approved Standard. Second edition, document M27-A2. National Committee for Clinical Laboratory Standards, Wayne, Pa., 2002.

8. Shin J.H., Og Y.G., Cho D., et al. Molecular epidemiological analysis of bloodstream isolates of Candida albicans from a university hospital over a five-year period. J Microbiol 2005;43:546-54. 
9. Huang Y.C., Lin T.Y., Leu H.S., Wu J.L., Wu J.H. Yeast carriage on hands of hospital personnel working in intensive care units. J Hosp Infect 1998;39:47-51.

10. Bonassoli L.A., Bertoli M., Svidzinski T.I.E. High frequency of Candida parapsilosis on the hands of healthy hosts. J Hosp Infect 2005;59:159-62.

11. Levin A.S., Costa S.F., Mussi N.S., et al. Candida parapsilosis fungemia associated with implantable and semi-implantable central venous catheters and hands of health care workers. Diagn Microbiol Infect Dis 1998;30:243-9.

12. Sadfar A., Fine J.P., Maki D.G. Meta-analysis: methods for diagnosing intravascular device-related bloodstream infection. Ann Intern Med 2005;142:451-66.

13. Lupetti A., Tavanti A., Davini P., et al. Horizontal transmission of Candida parapsilosis candidemia in a neonatal intensive care unit. J Clin Microbiol 2002;40:2363-9.

14. Barchiesi F., Caggiano G., Di Francesco L.F., et al. Outbreak of fungaemia due to Candida parapsilosis in a pediatric oncology unit. Diagn Microbiol Infect Dis 2004;49:269-71.

15. Clark T.A., Slavinski S.A., Morgan J., et al. Epidemiologic and molecular characterization of an outbreak of Candida parapsilosis bloodstream infections in a community hospital. J Clin Microbiol 2004;42:4468-72.
16. Almirante B., Rodrý'guez D., Cuenca-Estrella M., et al. Epidemiology, risk ractors, and prognosis of Candida parapsilosis bloodstream ýnfections: case-control populationbased surveillance study of patients in Barcelona, Spain, from 2002 to 2003. J Clin Microbiol 2006;44:1681-5.

17. Bassetti M., Righi E., Costa A., et al. Epidemiological trends in nosocomial candidemia in intensive care. BMC Infect Dis 2006; 6:21 (http://www.biomedcentral.com/1471-2334/6/21).

18. Kuhn D.M., Chandra J., Mukherjee P.K., Ghannoum M.A. Comparison of biofilms formed by Candida albicans and Candida parapsilosis on bioprosthetic surfaces. Infect Immun 2002;70:878-88.

19. Ergon, M.C., Yucesoy M. Molecular epidemiology of Candida species isolated from urine at an intensive care unit. Mycoses 2005; 48:126-31.

20. Humhreys H. Does molecular typing make any contribution to the care of patients with infection? Clin Microbiol Infect 2004; $10: 269-71$.

21. McGinnis M.R., Nunn G.B. Sequence based fungal identification and classification. In: Molecular Microbiology Diagnostic Principles and Practice. ASM Press Washington DC, p: 561-8, 2004.

22. Yamamoto T., Ueta E., Kamatani T., Osaki T. DNA identification of the pathogen of Candidal aspiration pneumonia induced in the course of oral cancer therapy. J Med Microbiol 2005;54:493-6. 